\title{
Preliminary study for the detection of neutrons in heavy-ion collisions with charged particle detectors
}

\author{
L. Auditore ${ }^{1,2}$, A. Pagano ${ }^{3}$ and P. Russotto ${ }^{3}$ \\ for EXOCHIM COLLABORATION
}

${ }^{1}$ INFN, Gruppo Collegato di Messina, Messina, Italy

2 Dipartimento di Fisica e Scienze della Terra, Università di Messina,

Messina, Italy

${ }^{3}$ INFN, Sezione di Catania, Catania, Italy

\begin{abstract}
At Laboratori Nazionali del Sud (LNS) the CHIMERA $4 \pi$ multidetector has been designed and setup to detect charged particles emitted in heavy ion collisions at intermediate energies. Properties and performances of CHIMERA have been widely demonstrated by published results obtained in the performed experiments. Moreover, in recent years, a new charged particle detector (ChPD) for correlation studies (FARCOS) has been designed, and recently a first prototype has been coupled to CHIMERA, in order to test performances in view of correlation measurements in coincidence with $4 \pi$ detectors. Simultaneous neutrons and charged particles detection in heavy ion collisions represents an important experimental progress for future experiments to be performed with both stable and exotic nuclei. In order to investigate about this possibility, simple Monte Carlo simulations have been performed. Preliminary simulations have been carried out by means of MCNPX transport code to evaluate the perturbation effects, including cross-talk and time response, induced in CHIMERA and/or FARCOS Si-CsI(Tl) telescopes on (typical $20 \mathrm{MeV}$ ) neutron signals coming from a typical reaction in heavy ion collisions at the Fermi energy. Moreover, first data analysis results of the INKIISSY experiment indicates sizable probability to detect neutrons by properly shadowing CHIMERA $\mathrm{Si}-\mathrm{CsI}(\mathrm{Tl})$ telescopes. Analysis is still in progress.
\end{abstract}




\section{Introduction}

Neutron detection in heavy ion collisions is an important tool to get complete information on the emitting source [1]. Depending on the reaction mechanism, neutrons carry part of teh information on the early dynamical phase of the reaction and the Equation of State (EoS). In particular, their detection contributes to better understand the asymmetry energy term of EoS [2].

Different experiments have been carried out to simultaneously detect charged particles and neutrons, thus pointing out the existing experimental problems related to the different techniques required for the simulataneous detection of neutral and charged particle [3-4].

At Laboratori Nazionali del Sud (LNS), the $4 \pi$ mutidetector CHIMERA has been operating for more than a decade in the framework of heavy ion collisions and its properties and performances have been widely demonstrated [5]. Moreover, a new detector, FARCOS [6], has been recently designed, assembled and tested, for particle correlation studies.

Both of them, whose structures are shown in Fig.1, if implemented for neutron detection will allow to obtain complete information on the reaction mechanisms.

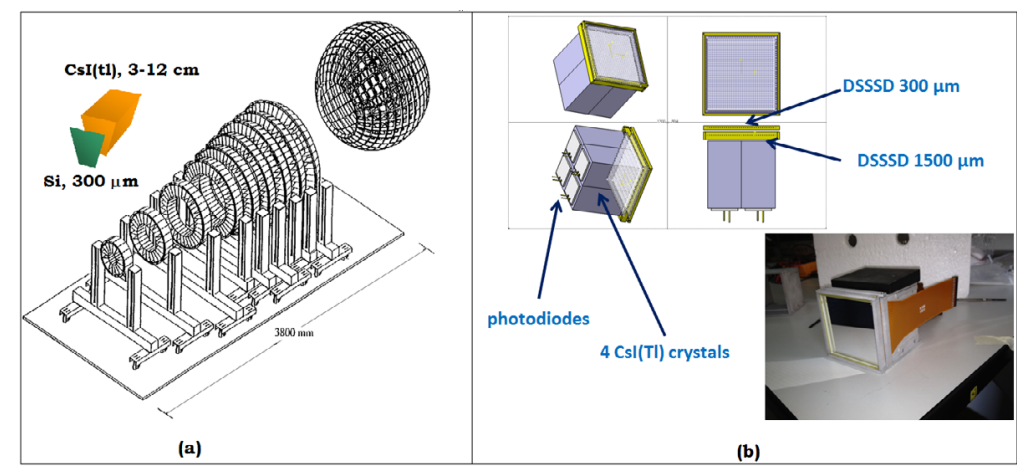

Figure 1: (a) CHIMERA; (b) FARCOS.

\section{Preliminary MC simulations and validation}

To the aim to detect neutrons with CHIMERA and/or FARCOS detectors, preliminary Monte Carlo simulations have been carried out with MCNPX code [7], thus evaluating the perturbation effects experienced by neutrons 
when punching through $\Delta \mathrm{E}-\mathrm{E}$ telescopes, i.e., background and cross talk effects.

Moreover, in order to validate MCNPX simulations the experimental setup

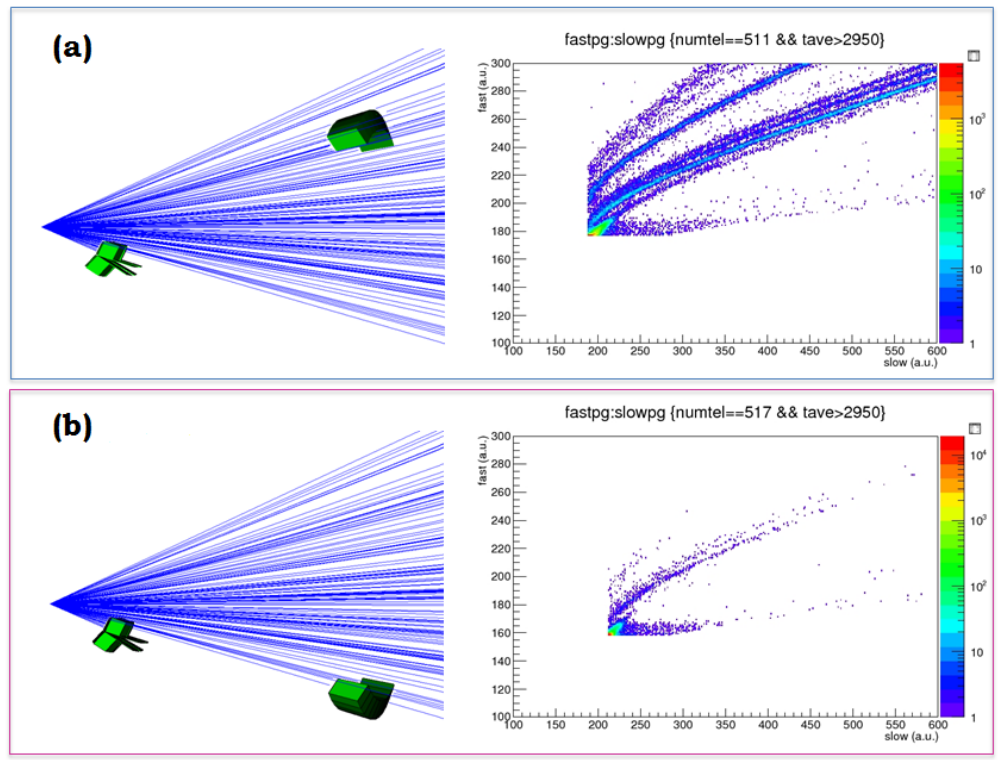

Figure 2: Comparison among Fast-Slow analysis for detectors n.511 (not covered by FARCOS) and n. 517 (covered by FARCOS).

used in the INKIISSY experiment carried out at LNS [8] has been simulated. During the INKIISSY experiment, FARCOS has been coupled to CHIMERA for the study of the reaction ${ }^{124} \mathrm{Xe}+{ }^{64} \mathrm{Zn} @ 35 \mathrm{AMeV}$.

In this configuration, FARCOS shielded the rings no. 8 and 9 of CHIMERA. Only neutrons (and gammas) can reach these detectors, the charged particles being stopped by FARCOS.

As a consequence, charged particle signals in $\mathrm{CsI}(\mathrm{Tl})$ detectors in the shadow beyond FARCOS are due only to neutrons.

A comparison among the experimental Fast-Slow analysis for detectors out and in the shadow of FARCOS is shown in Fig.2 while a preliminary comparison among the theoretical and experimental spectra of charged particles $(\mathrm{p}, \mathrm{d}, \alpha)$ as detected in the $\mathrm{CsI}(\mathrm{Tl})$ which are in the shadow beyond FARCOS is shown in Fig.3.

Information on the probable neutron energy and angular spectra have been obtained from the energy and angular spectra of protons emitted in the re- 
action ${ }^{124} \mathrm{Sn}+{ }^{64} \mathrm{Ni} @ 35 \mathrm{AMeV}$, studied at LNS [9-11].

Some discrepancies can be observed and work is in progress to better understand their origin and, consequently, to better adapt the simulations to the experimental setup.
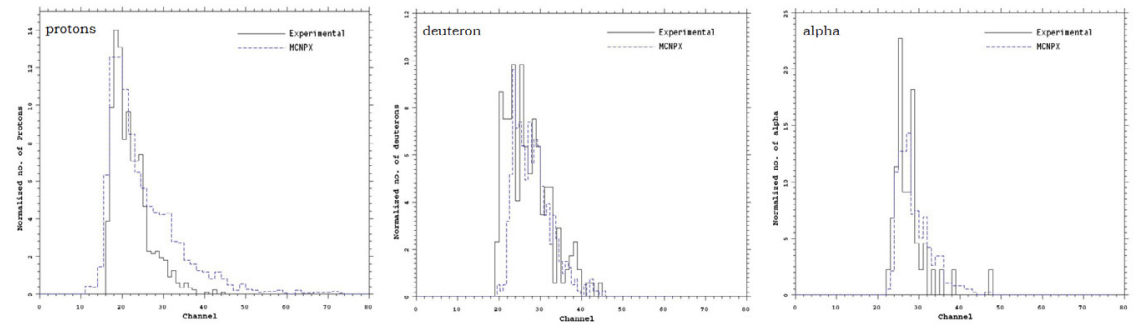

Figure 3: Comparison among MCNPX and experimental energy spectra of charged particles detected in a $\mathrm{CsI}(\mathrm{Tl})$ covered by FARCOS.

\section{Conclusions}

The EXOCHIM collaboration is working to study the possible implementation of simultaneous neutron and charged particle detection with CHIMERA and FARCOS. First preliminary experimental data, as compared with MC simulations, seem to indicated good perspectives for future upgrades.

\section{Bibliography}

[1] Ghetti R. et al., Nucl.Phys. A, Vol. 660:(1), (1999) 20.

[2] L.W. Chen et al., Phys. Rev. C68, (2004) 014605.

[3] W. Trautmann et al., Proccedings of Science, XLIX International Winter Meeting on Nuclear Physics, 24-28 Jan 2011, Bormio, Italy.

[4] P. Marini et al., Nucl Instr. Meth. A 707 (2013) 80.

[5] E. De Filippo, A. Pagano, EPJ A 50 (2014) 32.

[6] E.V. Pagano, IWM-EC2014, May 6-9, Catania, Italy,

[7] D. B. Pelowitz, ed., MCNPX User's Manual, Version 2.5.0, Los Alamos

National Laboratory report LA-CP-05-0369 (April 2005).

[8] Russotto P. et al, J. of Phys.: Conf. Series 515 (2014) 012020

[9] E. De Filippo et al., Phys. Rev. C 71 (2005) 044602.

[10] E. De Filippo et al., Phys. Rev. C 71 (2005) 064604.

[11] P. Russotto et al., Phys. Rev. C 81 (2010) 064605. 